\title{
A review of the safety of niclosamide, pyrantel, triclabendazole and oxamniquine
}

\author{
D. Ofori-Adjei ${ }^{\text {a }}$, A.N.O. Dodoo ${ }^{\mathrm{a}, *}$, A. Appiah-Danquah ${ }^{\mathrm{a}}$ and M. Couper ${ }^{\mathrm{b}}$ \\ ${ }^{a}$ Centre for Tropical Clinical Pharmacology \& Therapeutics, University of Ghana Medical School, \\ Accra, Ghana \\ ${ }^{\mathrm{b}}$ PSM, World Health Organisation, Geneva, Switzerland
}

\begin{abstract}
Detailed safety review of the intestinal anthelminthics (pyrantel and niclosamide) and antischistosomal and antitrematodes (oxamniquine and triclabendazole) was conducted using publications in journals and major reference sources combined with the assessment of adverse drug reaction (ADR) reports in the database of the WHO Collaborating Centre for International Drug Monitoring in Sweden. Data from available limited publications on the subject provide evidence in support of the overall safety of these medicines with no publication citing an unfavourable safety assessment of any of the drugs. Whilst there have been concerns about the risk of seizures with oxamniquine, these concerns have not diminished the extent to which the medicine has been used in several countries. All four medicines appear to be safe and well tolerated even when used in mass treatment campaigns in several countries. This safety review indicates that oxamniquine, triclabendazole, niclosamide and pyrantel have favourable benefit-risk profiles for use in humans. However, in order to enhance the evidence base to determine safety issues in relation to medicines that are used heavily in settings with poor or non-existent pharmacovigilance systems, it is recommended that the WHO pushes its current supportive efforts for the setting up of such systems globally, especially in resource-limited countries. The four drugs are all retained on the current list of the WHO Model List of Essential Medicines.
\end{abstract}

Keywords: Niclosamide, pyrantel, triclabendazole, oxamniquine, safety review, pharmacovigilance

\section{Introduction}

Parasitic infections are a major cause of disease burden, particularly in many countries in the tropics and sub-tropics. Several millions of people suffer because of diseases caused by schistosomes and other trematodes, and soil-transmitted helminths. The importance of schistosomiasis and soil-transmitted helminthsic infections have been recognised as priority health problems and through guidelines and technical assistance the WHO has encouraged member countries to set up control programmes [21]. At the 54th World Health Assembly a resolution expressing concern about the global impact of soil-transmitted helminthiasis and schistosomiasis was adopted. Periodic drug administration is one of the three principal strategies for reducing the burden of these diseases. The others are health education and information campaigns and the augmentation of knowledge and skills with regard to infections in the public health system at central and peripheral levels.

\footnotetext{
*Address for correspondence: Dr. Alexander Nii Oto Dodoo, Centre for Tropical Clinical Pharmacology \& Therapeutics, University of Ghana Medical School, P.O. Box GP 4236, Accra, Ghana. Tel.: +233244255064; Fax: +23321668219; E-mail: anododoo@ug.edu.gh.
} 
This review examines the safety of oxamniquine, triclabendazole, niclosamide and pyrantel as used in humans. It must be noted that these drugs have been used extensively in veterinary practice but the review focuses only on safety in humans.

\section{Method}

\subsection{Data sources}

A search was made on PUBMED and "Google Scholar" (http://scholar.google.com; sites accessed in January 2005) for all four drugs with emphasis on publications with the keywords "safety", "adverse reactions", "adverse drug reactions", "drug effect", "adverse effects" or "clinical trials". The Cochrane database was also inquired for reviews on any of the four medicines. Following the electronic web searches, copies of available texts were obtained from the local library or electronically through HINARI for free and/or preferential access to selected online journals. In addition, through HINARI, specific high profile journals on infectious diseases and parasitology were searched for publications on the four medicines. The search of the primary literature was complemented with review of textbooks and reference material including the WHO Model Formulary 2004, the British National Formulary (47, 2004), USP DI (2004), Martindale - the Extra Pharmacopoeia (31st edition, 1996), Meyler's Side Effects of Drugs (12th edition, 1992) and Stockley's Drug Interactions [23]. Adverse events information received from this desk review was added to those retrieved from the UMC database and reviewed together. All the information retrieved were reviewed and summarised by a clinical pharmacologist and a pharmacist (both with expertise in pharmacovigilance). An updated search of the WHO database and the primary literature was carried out again in May 2006 to update the information contained herein in preparation for submission of the manuscript for publication.

\subsection{Limitations}

This review was limited by the number of reports and journal articles that contain information on adverse events for most of the anthelminthics. Although the burden of the diseases lies predominantly with developing countries, there were very few reports and articles from the literature except for countries in South America. Most of the reports were dated before the availability of electronic versions of the publications on databases and hard copies were not easy to obtain. Furthermore, since most of the countries where these drugs are widely deployed do not have any formal pharmacovigilance systems, they do not contribute to the global database of adverse drug reactions maintained at the Uppsala Monitoring Centre (the UMC). Hence, any analysis of adverse events reported to the WHO database on these drugs gives only a partial picture. Furthermore, ADR reports in the WHO database were used to give indications of safety of individual compounds without case causality assessment of individual cases. The association between ADRs and these cases will clearly vary from "certain" through "uncertain" to "unclassifiable" thus their usage in this review is merely as "indicator" of safety with each medicine; using the extent of reporting as an estimate of safety: even then, there were very few reports making the need for detailed assessments of reports and possible data mining of doubtful scientific value. Finally, it was also not possible to get access to International Medical Statistics (IMS) data to determine the extent of current consumption of these products and to relate frequency of reported adverse reactions to levels of consumption. 


\section{Results}

The safety assessment of each of the four medications is presented below. Very few publications were found in the primary literature that provided detailed information on how safety of the medicines was assessed. Many of these studies made general statements regarding adverse/side effects without giving details of the procedures involved in determining or assessing safety.

\subsection{Niclosamide}

Niclosamide (2',5-dichloro-4'-nitrosalicylanilide; 5-chloro-N-(2-chloro-4-nitrophenyl)-2-hydroxybenzamide) is a nitrosalicylanilide introduced in early 1960s as an anticestodal drug. The drug has been used extensively since then and was listed in the WHO Model List of Essential Medicines 2003 and the WHO Model Formulary 2004. Due to safety and efficacy considerations, it is retained on the WHO Model List of Essential Medicines 2005 as well as the WHO Model Formulary 2006 (mednet3.who.int/) for treating Taenia saginata, T. solium, Hymenolepis nana and Diphyllobothrium latum infections.

Niclosamide is an anthelmintic which is active against most tapeworms, including the beef tapeworm (Taenia saginata), the pork tapeworm (T. solium), the fish tapeworm (Diphyllobothrium latum), the dwarf tapeworm (Hymenolepis nana) and the dog tapeworm (Dipylidium caninum).

\subsubsection{Adverse effects}

The adverse effect of niclosamide includes the following: nausea, retching, abdominal pain, lightheadedness and pruritus (WHO Model Formulary 2006). Since 1971, there have been 82 reports of suspected adverse drug reactions to niclosamide in the WHO database. These 82 reports from 16 countries involve 173 reactions from 16 countries (Fig. 1 and Table 2). The most common adverse reactions are those involving the skin and appendages (43), gastrointestinal tract (38) and cardiovascular system (28). There were 9 reports of anaphylactic shock and anaphylactoid reactions (Tables 1 and 2). (UMC database accessed on 23/05/06.)

In a publication by Bagheri et al. of adverse drug reactions involving 9 subjects and reported between 1985 and 1999, the majority of the reports related to the use of the drug in taeniasis [3]. The ADRs occurring during treatment for taeniasis were abdominal pain and vomiting (3), dizziness (2) and polymorph erythema (1), sweating (1) and anaphylactoid reaction requiring hospitalization (1).

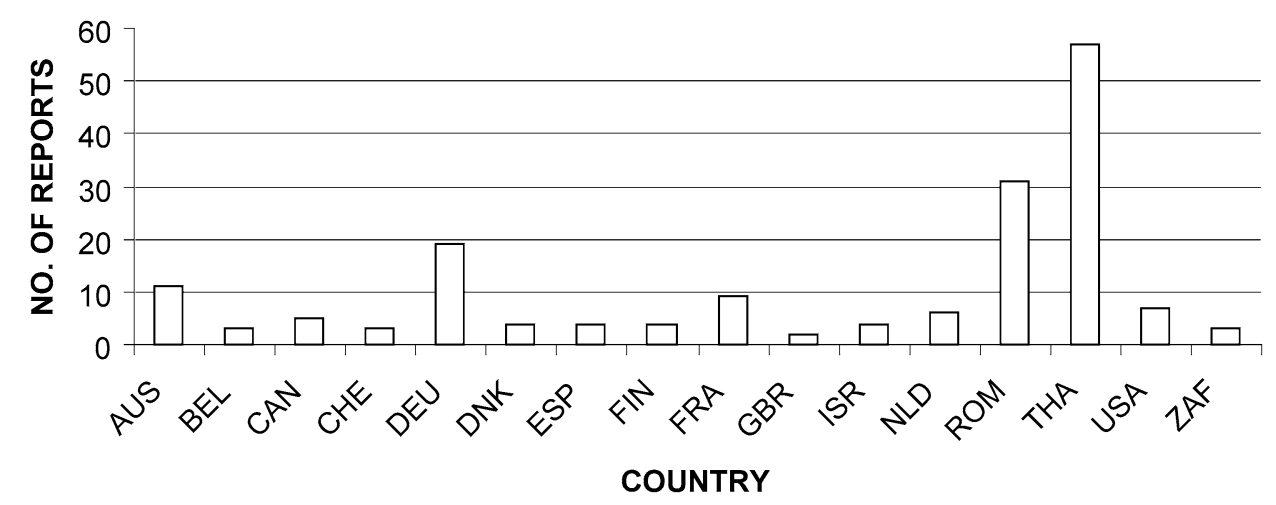

Fig. 1. ADRs on niclosamide in the UMC database. 
Table 1

Most common ADRs to niclosamide in the UMC database

\begin{tabular}{lc}
\hline Adverse drug reactions (ADRs) & Number of reports \\
\hline Abdominal pain & 15 \\
Anaphylactic stock & 5 \\
Cyanosis & 12 \\
Diarrhoea & 6 \\
Fatigue & 5 \\
Methaemoglobinaemia & 7 \\
Nausea & 6 \\
Pruritus & 8 \\
Rash & 11 \\
Sweating increased & 6 \\
Vomiting & 8 \\
\hline
\end{tabular}

Table 2

Adverse reactions to niclosamide in the UMC database

\begin{tabular}{lc}
\hline System organ & Number of reports \\
\hline Body as a whole - general disorders & 27 \\
Cardiovascular disorders, general & 16 \\
Centre and peripheral nervous system disorders & 12 \\
Foetal disorders & 2 \\
Gastro-intestinal system disorders & 38 \\
Heart rate and rhythm disorders & 5 \\
Liver and biliary system disorders & 4 \\
Poison specific terms & 1 \\
Psychiatric disorders & 4 \\
Red blood cell disorders & 7 \\
Resistance mechanism disorders & 1 \\
Respiratory system disorders & 8 \\
Skin and appendages disorders & 43 \\
Urinary system disorders & 2 \\
Vascular (extracardiac) disorders & 1 \\
White cell and res disorders & 1 \\
\hline
\end{tabular}

\subsubsection{Contra-indication}

Niclosamide is not contra-indicated for use in both small children and pregnant women. However, there is no data on its distribution into milk. Furthermore, there is no documented data on the effect of niclosamide on the elderly or its capacity to induce mutation or produce cancerous cells. Niclosamide is soluble in alcohol which enhances its absorption raising the possibility of dose related adverse effects. Alcohol intake is therefore forbidden during treatment with niclosamide.

\subsection{Oxamniquine}

Oxamniquine (1,2,3,4-tetrahydro-2-isopropylaminomethyl-7-nitro-6-quinolylmethanol) is a tetrahydroquinoline derivative used in the treatment of schistosomiasis caused by Schistosoma mansoni (acute stage and chronic hepatosplenic disease) but not by other species of Schistosoma. It is used in intestinal 
schistosomiasis due to Schistosoma mansoni (acute stage and chronic hepatosplenic disease). Oxamniquine is retained on the WHO Model List of Essential Medicines 2005 and the WHO Model Formulary 2006 as a complementary drug for treating intestinal schistosomiasis due to Schistosoma mansoni (acute stage and chronic hepatosplenic disease). The effective dose for oxamniquine varies geographically and there have been reports of resistance to oxamniquine [12].

\subsubsection{Adverse effects}

Adverse effects commonly associated with the use of oxamniquine include dizziness, drowsiness, headache, nausea, vomiting and diarrhoea (WHO Model Formulary 2006). These reportedly occur in up to a third of patients. The drug also causes intense reddish discolouration of urine [16]. Rare adverse events include urticaria, hallucinations, epileptiform convulsions, raised liver enzyme values; transient fever, eosinophilia and scattered pulmonary infiltrates (Loeffler syndrome) - after 3 day course in patients in Egypt and Eastern Mediterranean. It may also cause pruritic skin rashes.

In a Cochrane review, Saconato and Atallah [20] reported that no deaths were associated with oxamniquine use in the treatment of schistosomiasis caused by $S$. mansoni. They evaluated 372 patients and noted two cases of seizures, 63 cases of diarrhoea and 74 cases of abdominal pain. The review also compared the safety of oxamniquine and noted important differences in adverse effects. For example, diarrhoea and abdominal pain were reported nearly twice ( 38 of 544 patients $-7 \%$ and 115 out of 571 cases $20 \%$, respectively) in the oxamniquine group compared to the pyrantel group (74 out of 536 patients $14 \%$ and 240 out of 563 cases $-42 \%$, respectively). Myalgia on the other hand was reported by 7 of 352 patients $(2 \%)$ on oxamniquine though none of the 327 patients on pyrantel reported it. Seizures were only looked out for in the oxamniquine group where 2 out of 372 patients reported with this reaction.

Several adverse effects to oxamniquine are reported in the literature (Table 3). However, the study designs employed varied tremendously and there was very little indication on how safety data was collected. While the incidence of adverse events appears quite high, most of them (especially the gastrointestinal tract symptoms) appear to be mild. The reports of epileptic seizures or convulsions, however, indicate the need for caution in the use of the drug in susceptible patients. The high incidence of seizures means that the performance of skilled tasks e.g. driving and operating machinery, after intake of oxamniquine may be impaired.

Table 3

Incidence of adverse events to oxamniquine in the primary literature

\begin{tabular}{lcc}
\hline Adverse reaction & Incidence (\%) & References \\
\hline Dizziness & 40 & {$[12,24]$} \\
Vomiting & 1.1 & {$[24]$} \\
Diarrhoea & 14.7 & {$[24]$} \\
Abdominal pain or discomfort & 41.1 & {$[24]$} \\
Epileptic seizure & 2.4 & {$[24]$} \\
Reversible abnormal electroencephalograph & 20 (after 2 nd dose) & {$[24]$} \\
Auditory and visual hallucinations & $0.4-0.8$ & {$[24]$} \\
Headache or burning sensation & 3.2 & {$[24]$} \\
Itching & 7.4 & {$[24]$} \\
High pulse pressure and sweating & 6 & {$[24]$} \\
Seizure & 2.4 & {$[12,24]$} \\
Convulsions & $\sim 14$ out of 9 million & {$[12]$} \\
\hline
\end{tabular}


Table 4

Adverse reactions to oxamniquine in the UMC database

\begin{tabular}{lc}
\hline System organ & Number of reports \\
\hline Centre and peripheral nervous system disorders & 7 \\
Gastro-intestinal system disorders & 1 \\
Psychiatric disorders & 2 \\
\hline
\end{tabular}

In the WHO database 10 suspected adverse events have been reported since 1979. These reports were from Great Britain, Netherlands, Norway and USA and include convulsions (6), dizziness (1), glossitis (1), confusion (1) and somnolence (1) (Table 4). (UMC data accessed on 23/05/06.)

\subsubsection{Contra-indication}

Oxamniquine is contra-indicated in epilepsy. There is no data on distribution into milk but the WHO Model Formulary advices avoidance during lactation. No paediatric and geriatrics specific problems have been documented and the medicine may be used in these populations if the benefits far outweigh any unexpected risks. No carcinogenicity, maternal toxicity and teratogenicity were found in mice and rabbits in relation to ingestion of oxamniquine.

\subsubsection{Discussion}

Oxamniquine administration is accompanied by a relatively high incidence of minor gastrointestinal and central nervous system side effects. These are often mild and have not limited the widespread use of the drug. It is a useful alternative to praziquantel in areas where the latter is ineffective. Several studies have been carried out on the drug, especially on regional variations of effective dosages. Whilst resistance to oxamniquine has been reported [6] there is no evidence that its utility is waning. It has been used in mass campaigns and though minor side effects are frequent as stated previously, these appear not to have been a barrier to widespread use in all age groups and populations [12]. With increasing reports and concerns on development of resistance to praziquantel [7-10,15,22], the usefulness of oxamniquine as an alternative to praziquantel would remain paramount. The drug is very effective in treating S. mansoni infections and it is also a useful alternative in areas where praziquantel have already failed.

\subsection{Triclabendazole}

Triclabendazole (5-chloro-6-(2,3-dichlorophenoxy)-2-(methylthio)benzimidazole) is a benzimidazole anthelmintic. It is used as the primary agent in fascioliasis (Fasciola hepatica and Fasciola gigantica) and is the primary or alternate agent to praziquantel in paragonimiasis. It has no action against nematodes as do other benzimidazoles in clinical use. It is on present on the WHO Model List of Essential Medicines 2005 and WHO Model Formulary 2006 for use in fascioliasis and paragonimiasis.

\subsubsection{Adverse effects}

The only adverse effects of triclabendazole listed in the WHO Model Formulary 2006 are gastrointestinal discomfort and headache. The few studies available in the literature report that the drug is well tolerated and safe without providing specific information on adverse effects or how they were monitored in studies [1,19]. Gastrointestinal discomfort and headache have been particularly mentioned as side effects.

In a study on the use of triclabendazole in paragonimiasis (in comparison with praziquantel), the main side effects noted were dizziness, headache and abdominal pain. These occurred more frequently in those receiving praziquantel [4]. Nausea and vomiting occurred only in the group receiving praziquantel. No 
Table 5

Reported incidence of adverse events to triclabendazole

\begin{tabular}{lcc}
\hline Adverse reaction & Incidence $(\%)$ & References \\
\hline Dizziness & 5.5 & {$[4,5]$} \\
Headache & 0.5 & {$[4,5]$} \\
Abdominal pain & 5.0 & {$[4,5]$} \\
Fever & 2.0 & {$[4,5]$} \\
Diarrhoea & 1.0 & {$[4,5]$} \\
Jaundice & 0.7 & {$[11]$} \\
\hline
\end{tabular}

important changes in blood biochemistry were noted in the two groups. In a separate study in Cuba involving 82 patients ( 51 females, 31 males, age $15-81$ yrs, mean 42 yrs) with chronic or latent $F$. hepatica infection refractory to previous anti-helminthic chemotherapy in a 60-day open, non-comparative trial, a total of 74 adverse events (possibly related to therapy) were reported by 54 patients. The most common adverse event was colic-like abdominal pain in $40(49 \%)$ patients. This was related to the expulsion of the parasite through the bile duct. Fifty three of the adverse events were graded as mild, 20 as moderate, and only 1 as serious - a biliary colic which responded to treatment with a spasmolytic within two hours [18]. Chills, fever, leucopoenia and upper abdominal colic have been described in Meyler's Side Effects of Drugs though the incidence rate is not given. In the UMC Database, there are two reports of adverse reactions to triclabendazole. These were reported from Spain and Germany in 2001-2002. The two reports contained four adverse reactions - vomiting, jaundice, raised hepatic enzymes and psychosis (UMC data accessed on 23/05/06). These few reports of adverse reactions to triclabendazole mirror the information in the literature (Table 5).

\subsubsection{Contra-indication}

No contra-indications have been recommended from human studies and animal studies show no evidence of teratogenicity or embryocidal effects [25]. The drug is distributed in breast milk and there is no paediatric and geriatric specific problem.

\subsubsection{Discussion}

Triclabendazole appears to be a very well tolerated drug. The low number of reports in the WHO database could be a reflection of its safety though lack of pharmacovigilance systems in several of the countries where the drug is used may also be a factor. There are reports of the successful use of triclabendazole in treating praziquantel-ineffective fascioliasis [14,17]. It is the drug of choice for both fascioliasis and paragonimiasis. Fewer side effects were recorded with its use in comparison with praziquantel [4].

\subsection{Pyrantel}

Pyrantel (1,4,5,6-tetrahydro-1-methyl-2-[(E)-2-(2-thienyl)vinyl]pyrimidine 4,4'-methylenebis(3-hydroxy-2-naphthoate) is a pyridine derivative anthelmintic which appears to act by paralysing susceptible worms which are then dislodged by peristaltic activity. It is effective against intestinal nematodes including roundworms (Ascaris lumbricoides), threadworms (Enterobius vermicularis) and Trichostrongylus spp., the tissue nematode Trichinella spiralis, and hookworms. It is also used in treating mixed helminthic infections. Pyrantel may not be effective against all strains of a particular helminth. It is on the WHO Model List of Essential Medicines 2005 and the WHO Model Formulary 2006 for use in 
Table 6

Adverse reactions to pyrantel in the UMC database

\begin{tabular}{lc}
\hline System organ & Number of reports \\
\hline Body as a whole - general disorders & 67 \\
Cardiovascular disorders, general & 3 \\
Centre and peripheral nervous system disorders & 64 \\
Foetal disorders & 6 \\
Gastro-intestinal system disorders & 178 \\
Hearing and vestibular disorders & 2 \\
Heart rate and rhythm disorders & 4 \\
Liver and biliary system disorders & 9 \\
Metabolic and nutritional disorders & 2 \\
Musculo-skeletal system disorders & 8 \\
Platelet, bleeding and clotting disorders & 5 \\
Psychiatric disorders & 21 \\
Red blood cell disorders & 1 \\
Respiratory system disorders & 7 \\
Skin and appendages disorders & 56 \\
Urinary system disorders & 7 \\
Vascular (extracardiac) disorders & 4 \\
Vision disorders & 21 \\
White cell and res disorders & 3 \\
\hline
\end{tabular}

treating ascariasis, hookworm infections, enterobiasis and trichostrongyliasis as well as tissue nematode infections.

\subsubsection{Adverse effects}

Side effects associated with pyrantel are generally mild and include gastrointestinal disturbances, headache, dizziness, drowsiness, insomnia, rash and elevated liver enzymes. A review of the French Pharmacovigilance database revealed 10 ADR reports during treatment for ascariasis (4), pinworm (5) and an unspecified helminth infection [3]. The ADRs reported included four cases of nausea, vomiting and flatulence; pruritus and urticaria ( 2 cases); and a case each of dizziness, headache and hypotonia. One case of paraethesia associated with ataxia and weakness in a 32 year old man showed a positive rechallenge. Two hundred and three adverse drug reaction case reports involving 468 adverse reactions associated with pyrantel use are present in the WHO ADR database (Table 6). Majority of the reports originate from Australia a country with an active pharmacovigilance system. The main organs and systems affected are the gastrointestinal tract (178 reports), body as a whole (67 reports), central and peripheral nervous systems (64 reports) and the skin and appendages (56 reports). There are 21 reports each under psychiatric disorders and vision disorders calling for an urgent need to review patients on this drug closely for the manifestations of both psychiatric and visual disorders.

\subsubsection{Contra-indication}

Pyrantel is not recommended for use during pregnancy. It is poorly absorbed from the gastrointestinal tract and very low levels are detected in breast milk. There are no paediatric specific problems documented and the drug is not contraindicated for use in children less than 1 year of age.

It has been reported that piperazine may counteract the anthelmintic effect of pyrantel [2] and there is a single report of an interaction between pyrantel and theophylline leading to increase serum theophylline levels [13]. 


\subsubsection{Discussion}

There is extensive and established safety experience with pyrantel in several countries. The drug has been well studied and adverse reaction profiles documented. Even though there are several adverse reactions reported in association with its use in the WHO database, the drug is still widely used for hookworm infections, enterobiasis and tissue nematode infections. The most frequent adverse reactions to pyrantel relate to the gastrointestinal system and are mild. There is no information to suggest an unfavourable safety profile of the drug under current conditions of use and the drug remains a safe and useful anthelmintic drug that has a place in the management of nematode infections.

\section{Conclusions}

Niclosamide is a well established drug which has been used in several countries since 1960. Whilst the absence of formal pharmacovigilance systems in most of the countries where the drug has been widely deployed limits the safety data available for analysis, anecdotal evidence and the published literature points to very few adverse events in its usage. If there are substantial safety concerns associated with the use of niclosamide, these will have been noted during its $40+$ years of usage. The current review supports the continuous use of niclosamide as no major safety concerns have been raised. Its safety in children and pregnant women and efficacy and safety in treating T. solium carriers even in the presence of (neuro) cysticercosis is an advantage over praziquantel. Oxamniquine administration is accompanied by a relatively high incidence of minor gastrointestinal and central nervous system side effects. These are often mild and have not limited the widespread use of the drug. The drug is very effective in treating S. mansoni infections. It is also a useful alternative in areas where praziquantel have failed. Triclabendazole is a well tolerated drug with minimal safety concerns. Pyrantel remains a safe and useful anthelmintic drug that has a place in the management of nematode infections. All four drugs are present on the WHO Model List of Essential Medicines 2005 and the WHO Model Formulary 2006 and expert opinion indicate that on the basis of currently available information, these drugs are safe for the conditions wherein their use is indicated.

\section{Acknowledgement}

This work was carried out as APW for WHO-HQ/PSM by David Ofori-Adjei.

\section{References}

[1] W. Apt, X. Aguilera, F. Vega, C. Miranda, Y. Zulantay, C. Perez, M. Gabor and P. Apt, Treatment of human chronic fascioliasis with triclabendazole: drug efficacy and serological response, Am. J. Trop. Med. Hyg. 52(6) (1995), 532-535.

[2] M.L. Aubury, P. Cowell, M.J. Davey and S. Shevde, Aspects of the pharmacology of a new anthelmintic pyrantel, $B r$. J. Pharmacol. 38 (1970), 332-344.

[3] H. Bagheri, E. Simiand, J.L. Montastruc and J.F. Magnaval, Adverse reactions to anthelmintics, Ann. Pharmacother. 38(3) (2004), 383-388.

[4] M. Calvopina, R.H. Guderian, W. Paredes, M. Chico and P.J. Cooper, Treatment of human pulmonary paragonimiasis with triclabendazole: clinical tolerance and drug efficacy, Trans. Roy. Soc. Trop. Med. Hyg. 92(5) (1998), 566-569.

[5] M. Calvopina, R.H. Guderian, W. Paredes and P.J. Cooper, Comparison of two single-day regimens of triclabendazole for the treatment of human pulmonary paragonimiasis, Trans. Roy. Soc. Trop. Med. Hyg. 97(4) (2003), 451-454.

[6] D. Cioli, L. Pica-Mattoccia and S. Archer, Drug resistance in schistosomes, Parasitol. Today 9(5) (1993), 162-166.

[7] G.C. Coles, Drug resistance or tolerance in schistosomes?, Trends Parasitol. 18(7) (2002), 294. 
[8] G.C. Coles, W.T. Mutahi, G.K. Kinoti, J.I. Bruce and N. Katz, Tolerance of Kenyan Schistosoma mansoni to oxamniquine, Trans. Roy. Soc. Trop. Med. Hyg. 81(5) (1987), 782-785.

[9] A. Danso-Appiah and S.J. De Vlas, Interpreting low praziquantel cure rates of Schistosoma mansoni infection in Senegal, Trends Parasitol. 18 (2002), 125-129.

[10] M.J. Doenhoff, J.R. Kusel, G.C. Coles and D. Cioli, Resistance of Schistosoma mansoni to praziquantel: is there a problem?, Trans. Roy. Soc. Trop. Med. Hyg. 96 (2002), 465-469.

[11] H. El-Morshedy, A. Farghaly, S. Sharaf, L. Abou-Basha and R. Barakat, Triclabendazole in the treatment of human fascioliasis: a community-based study, East Mediter. Health J. 5(5) (1999), 888-894.

[12] R. Foster, A review of clinical experience with oxamniquine, Trans. Roy. Soc. Trop. Med. Hyg. 81 (1987), 55-59.

[13] L. Hecht, W.E. Murray and S. Rubenstein, Theophyline-pyrantel pamoate interaction, DIP Ann. Pharmacother. 23 (1989), 258.

[14] Y. Ishii, F. Nakamura-Uchiyama and Y. Nawa, A praziquantel-ineffective fascioliasis case successfully treated with triclabendazole, Parasitol. Int. 51(2) (2002), 205-209.

[15] N. Katz, R.S. Rocha, C.P. de Souza, P. Coura Filho, J.I. Bruce, G.C. Coles and G.K. Kinoti, Efficacy of alternating therapy with oxamniquine and praziquantel to treat Schistosoma mansoni in children following failure of first treatment, Am. J. Trop. Med. Hyg. 44(5) (1991), 509-512.

[16] J.L. Lefrock and B.R. Smith, Treatment of helminthic diseases, Am. Fam. Phys. 32(3) (1985), 182-188.

[17] J. Merino Alonso, M.J. Amerigo Garcia, L. Alvarez Rubio and I. Erdozain Ruiz, Human fascioliasis with atypical severe presentation, treatment with triclabendazole, Enferm. Infect. Microbiol. Clin. 16(1) (1998), 28-30.

[18] J.C. Millan, R. Mull, S. Freise and J. Richter (Triclabendazole Study Group), The efficacy and tolerability of triclabendazole in Cuban patients with latent and chronic Fasciola hepatica infection, Am. J. Trop. Med. Hyg. 63(5/6) (2000), 264-269.

[19] C. Ripert, B. Couprie, R. Moyou, F. Gaillard, M. Appriou and J. Tribouley-Duret, Therapeutic effect of triclabendazole in patients with paragonimiasis in Cameroon: a pilot study, Trans. Roy. Soc. Trop. Med. Hyg. 86(4) (1992), 417.

[20] H. Saconato and A. Atallah, Interventions for treating Schistosomiasis mansoni, Cochrane Review, The Cochrane Library, Issue 4, John Wiley \& Sons, Ltd., Chichester, UK, 2004.

[21] L. Savioli, D.A.P. Bundy and A. Tomkins, Intestinal parasitic infections: a soluble public health problem, Trans. Roy. Soc. Trop. Med. Hyg. 86 (1992), 353-354.

[22] F.F. Stelma, S. Sall, B. Daff, S. Sow, M. Niang and B. Gryseels, Oxamniquine cures Schistosoma mansoni infection in a focus in which cure rates with praziquantel are unusually low, J. Infect. Dis. 176(1) (1997), 304-307.

[23] I.H. Stockley (ed.), Drug Interactions, 4th edn, Pharmaceutical Press, London, 1996.

[24] K. Taddese and Z.A. Zein, Comparison between the efficacy of oxamniquine and praziquantel in the treatment of Schistosoma mansoni infections on a sugar estate in Ethiopia, Ann. Trop. Med. Parasitol. 82(2) (1988), 175-180.

[25] H. Yoshimura, Teratogenic evaluation of triclabendazole in rats, Toxicology 43(3) (1987), 283-287. 